\section{HEALER CHOICE IN MEDICALLY PLURALISTIC CULTURAL SETTINGS: AN OVERVIEW OF NEPALI MEDICAL PLURALISM}

\author{
Madhusudan Sharma Subedi*
}

\section{Introduction}

Cross-cultural comparison of medical setting around the world led to the formulation of general models of the relationships between the various medical traditions within single settings. The study of human confrontation with disease and illness, and of the adaptive arrangements made by human groups for dealing with ever-present danger has come to be a special branch of anthropology called medical anthropology. It is one of the youngest and most dynamic of the various branches of anthropology. It concerns with a wide variety of health related issues, including the etiology of the disease, the preventive measures that humans as members of socio-cultural systems have constructed or devised to prevent the onset of the diseases,

\footnotetext{
Mr. Subedi is an anthropologist and has been associated with Central Department of Sociology/Anthropology in the capacity of Part-time Lecturer.

The information used in this paper were collected in the year JulyDecember 1999 for my M. Phil. thesis in Social Anthropology, University of Bergen, Norway, and January -March 2002 for personal research. 1 am grateful to Norwegian Agency for International Development and Cooperation (NORAD) for financial support. I would like to thank Prof. Gunnar Haaland, University of Bergen for his comment on earlier draft of this paper.
}

and the curative measures that they have created in their efforts to eradicate the diseases or at least to mitigate its consequences.

Recently there has been a surge in publications, which address the importance of incorporating the 'body' in theories about the world of 'social reality'. In a given individual or social network, people's understandings of how their body functions influence health practices, symptom evaluation, and taking remedial actions. Help may be sought from a number of sources in the patient's social network, including friends, family, traditional healers, and the professionals (Christakis et al. 1994; Subedi 2001a). This is connected with a growth of medical anthropology and its interest in exploring the interface between natural processes, which affect the bodies we 'are living in' and socio-cultural processes in terms of which 'people understand and cope with the health of their bodies'. Our survival as individual human beings depends on natural processes maintaining the health of our bodies. These processes involve an interaction between natural processes internal to the body organism and processes involved in the physical interaction of our bodies with a natural environment. Like for any other species these processes are subject to 'natural law'. However, in contrast to other species, humans are not endowed with a genetically transmitted repertoire of behaviors, which allow a viable interaction in a natural environment. The genetically transmitted repertoires have been softened and weakened to such an extent that human beings neither orient themselves in their world nor communicate with each other without acquiring a great deal of knowledge through learning (Elias, 1991). In Geertz's words, "man is an animal suspended in webs of significance he himself has spun (1973:5)." These webs are clarified through elaborate symbolic exposition.

Without growing up in a context of cultural webs to interpret the interaction between the embodied self and its natural and social environment, the human animal is impossibility. In other words, humans are situated in an environment that entails both a natural dimension and a culturally cønstructed one. This "social environment" is an intrinsic system of interaction between nature and culture, which 
is created under the specific physical limits and imposes various material constraints upon human population. As humans we can only experience nature as we culturally construct it, imbue it with meaning, and interact with it in ways that fit within our particular cultural frame of understanding and emotion (Baer et al., 1997:39).

In this context the most important aspect of this cultural breakthrough is that it implies self-consciousness ability to see one's embodied self in the perspective of the unavoidability of bodily death. It seems reasonable to assume that the human consciousness of death makes the health of the human body a fundamental concern everywhere, and that this as the general starting point for my description and analysis of the way people in Nepal, cope with health in their natural and social environments.

The impact of a multiple tradition of medicine on health services remains an area little understood in Nepal. Hence, in determining the factors affecting the different types of healthcare traditions within a medically pluralistic cultural setting, it is essential to understand how the presence of medical pluralism may be related to or affect the choice and decision to seek different health-care services. I argue that the research on explanation of illness causality sheds light on health care behavior. Examinations of etiologies entail an exploration of folk epidemiology, factors which lead to vulnerability and susceptibility, as well as what can be done to avoid the risks.

\section{Methods and Materials}

In order to understand the events of health seeking behavior, I have reviewed Nepali medical pluralism and observed healing and referral process in Kathmandu Valley. I found it necessary to place them in the context of wider traditions of knowledge and institutional arrangements they are embedded in. Specifically, I have focused this study to provide the answers to the following questions:

How do the people in Nepal understand and create meanings on their bodily afflictions?
How do they relate their health problems with social and natural environments?

What sorts of range of therapeutic traditions are available and practiced in Kathmandu Valley?

What are the main causes behind the acceptance of different medical traditions?

What are the methods used by healers in their healing practices in Kathmandu Valley?

How and why are the choices made between and within the various options?

The research I describe here is designed to explore the systematic patterns of healers and laypersons concerning illness categories in relation to actual behaviors involving those illness and help seeking behaviors in Kathmandu Valley, where I intended to study the diverse healing practices from the point of view of both healers and laypersons. The basic method of data collection for this study was observation along with formal and informal discussions with healers as well as patients. Interviews with different healers took place at the medical shops, health posts, doctor's private clinics (both allopathic medicine and acupuncture therapy), and in the home of those (local healers both male and female) with whom I talked. I also discussed formally or informally with different people at teashops, informal gatherings and houses of different informants to understand the lay perception towards different medical traditions as well as within the medical tradition.

Several chains of subjects were developed simultaneously and I followed up those chains that widened the demography. I often followed a sequence of contacts, beginning with local administrators, local health authorities, and the other leaders in the political and administrative hierarchy. The number of conversations and other contacts with these informants varied greatly, depending on their availability, their breadth of information, and willingness to spend many hours with me. My argument here is that the part of people's ideas in terms of which they explain and handle can be seen as their theory of illness. I wanted to know how people (patients as well as healers) describe their health. For example, what are the locally defined illnesses? 
What are thought to be the causes and the degree of severity and symptoms? What treatments are sought? From whom? I also wanted people to talk about their explanation for their state of health; what they identify about themselves or their physical or social environments as important, and what, if anything, they believe can or should do to protect or enhance their health.

One further interesting venue of research was the attitude of different healers towards the other healing practices and the extent, which the therapy traditions overlap or diverge. My intention was to focus on the interaction of healers and the patients, and how they deal with the different cases of illness they are confronted with.

\section{Theoretical Framework}

People construct multiple and discrepant worlds by means of different traditions of knowledge available to them. We need to discover how much the different constructions are in fact distributed in their action and interactions (Barth 1993:271). Following this idea, I have developed my conceptual framework. Understanding of Bodily Afflictions and Explanatory Models

Human illness is not only a physical condition but a symbolic one as well. What we experience as health or illness is based on the perceptual judgements we make about the relative quality of our physical and psychological condition. The meanings individuals assign to their health status are strongly influenced by their cultural backgrounds and experiences. These culturally based meanings strongly influence the health care choices and decisions they make, their relative confidence in their health care providers and the treatment regimen, and even their actual physical responses to health care treatment.

In understanding bodily afflictions, it is useful, in my view, to consider the types of questions that people ask themselves when they feel unwell, or when they experience any sudden, unexpected events in their daily lives. My theoretical framework, in this thesis, is mostly based on people's understanding of the culturally constructed reality. I argue that the reality can only be fully understood by examining the specific contexts in which an ill person's socio-economic organizations and dominant worldviews are patterned. Kleinman (1980) has suggested a useful way of looking at the process by which illness is patterned, interpreted and treated, which he terms the 'Explanatory Models'. This is defined as the notions about an episode of illness and its treatment that are employed by all those engaged in the healing process. Both patients and practitioners hold explanatory models, and they offer explanations of illness and treatment to guide choices among available therapies and therapists and to cast personal and social meaning on the experience of illness. The explanatory model for a particular illness consists of (i) signs and symptoms by which the illness is recognized; (ii) presumed causes of illness; (iii) recommended therapies, (iv) the pathophysiology of the illness; and (v) prognosis (Kleinman, 1980:105-7).

As Kleinman points out, individuals are likely to have quite vague and indefinite models of explanations for their illnesses, depending on past experiences of the patients and his/ her circle of kin and friends. This tendency in research arose, in part, in relation to the growth of cultural pluralism, especially in matters of health and illness (Pelto and Pelto, 1996).

\section{Knowledge and Concerns}

Knowledge in itself is the result of a learning process that is strongly influenced by a number of factors. Once internalized this knowledge becomes belief. Each medical tradition provides a range of beliefs from which people make their reality and the culturally constructed reality. Healers and lay belief about health and ill-health may differ widely, and these influence the types of conditions that people bring to healers, how they present themselves to the healers, the types and quality of treatment that they are given. A major emphasis here is on exploring the variation within both lay and professional views. Connected to those considerations of knowledge and concerns, I have used Barth's (1993:5) idea of variation to make a sense of it in people's health seeking behavior as:

- $\quad$ There are variations in the definition of illness, and each medical tradition provides a unique cause and treatment for a distinctive set of illnesses. Thus the over- 
generalization is perhaps the most serious and frequent weakness of research on health seeking behavior.

- There are variations in level of "expertise" within the same medical tradition in the population. There is no identical, highly rigid pattern of the expertise. That is the interpretation given concerning the causes of illness and medicine prescribed varies within the same medical tradition.

These variations are important for better understanding of the lives of people, through processes involving those people's own ideas and activities. The different styles of knowledge transmission generate deep differences in the form, scale, and the distribution of the knowledge (Barth, 1990:640). The knowledge that is acquired, retained, and transmitted contains the key to explaining variations.

\section{The Arts of Impression Management}

In each medical tradition, we can see the interaction as a "performance", shaped by the environment and audiences, constructed to provide others with "impressions" that are consonant with the desired goals of the actor (Goffman, 1959:17). Each healer promotes the psychological excitement for a realization of a goal. In this way, a healer develops an identity or persona as a function of interaction with others through an exchange of information that allows for a more specific definition of identity and behavior. There is often a tendency for all types of healers to try to present themselves in such a way as to impress their patients in a socially desirable way. Thus impression management has, in my view, considerable implications for areas of health and healing.

\section{Medical Anthropology}

Health and ill-health are biological phenomena but their distribution and scale in particular populations are affected by the knowledge (including value pattern, technology, and view of the universe) and the social ties in terms of which people interact with their natural habitat. In other words, there is an intimate linkage between disease, medicine and human culture. In part, cultures are the responses made by human groups in the path of successful adaptation. Conceptualization of disease, including etiology diagnosis, prognosis or cure, all are part of the cultural repertory and equipment of human beings. Various conceptualizations of ill-health or disease can consequently not be understood apart from an understanding of the culture and social structure of groups holding them (Blustain, 1976; Helman, 1996; Kleinman, 1980; Stone, 1976; Leslie, 1978; 1980; Justice, 1986; Levitt, 1988; Subedi, 1989; DurkinLongley, 1984; Streefland, 1985; Dixit, 1999).

Medical anthropology looks at cultural conceptions of the body, health and illness. It also focuses on health behavior as a way to learn about social values and social relations. In western research traditions, medical sciences have made important contributions to understanding of the natural laws, which regulate the operation of these processes. A vast institutional complex has emerged directed towards further development of the medical research tradition (often referred to as biomedicine, allopathic medicine, modern medicine, western medicine or cosmopolitan medicine) and training of practitioners qualified to prescribe treatment to health seeking clients. The western medical tradition involves both knowledge about how the general environmental interactions (for example, food habits and hygienic practices) affect the body, and knowledge about how a specific treatment (prescription of medicines and surgical operations) may counteract particular disease. The disciplinary fields include genetic, paleopathology, epidemiology, nutrition and demographic anthropology.

Ethnomedical studies of health and healing are a second major emphasis in medical anthropology. This approach often attempts to discover the insiders' viewpoints in describing and analyzing health and systems of healing. Among the topics studied in this field are ethnoscience, ethnopharmacology, shamanism, and use of alternative therapies, medical pluralism and others. Third, many anthropologists focus their work on social problems and carry out interventions through applied medical anthropology. Among the areas of this subfield are studies of addictions, disabilities, and mental health issues; 
public health and family planning; clinical anthropology and health care delivery in pluralistic setting.

Whatever their disciplinary orientation, whether biomedicine, ethnomedicine, or policy and intervention work, many medical anthropologist agree that the concept of adaptation, defined as change and modification that enable a person or group to survive in a given environment, is a core theoretical construct of the field (McElroy and Townsend, 1996). The application of medical anthropology have contributed much to our understanding of the mechanism by which modern medicine and health programs are introduced into, get accepted or get rejected by different societies. The medical anthropological literature abounds with the theoretical discussion and case studies on the persistence of traditional medical practices and practitioners even in the presence of modern medicine, description of traditional medical systems in pluralistic medical setting.

By the early 1960s, the term medical anthropology has come to be used increasingly by anthropologists working in and around problems in health and disease in human societies. Therefore, it is not surprising that an anthropological study of health and occurrence and means of coping with disease can involve one deeply in the manner in which people perceive their world, in the characteristics of the human social system and in social values (Good, 1994). In this perspective, medical anthropology is not only a way of viewing the state of health and disease in society, but a way of viewing society itself (Lieban, 1977). One can not really understand how people react to illness, death or other misfortunes without understanding the type of culture that they have grown up in, or acquired -that is, of the lens through which they are perceiving and interpreting their world. In addition to the study of culture, it is also necessary to examine the social organization of health and illness in that society (the health care traditions) which includes the ways in which people have become recognized as ill, the way that they present their illness to other people, the attributes of those they present their illness to, and the ways that the illness is dealt with.
The particular tradition of western medical knowledge and its accompanying health care tradition represent one way of dealing with illness. Other societies have developed different understandings with different types of healers and medical traditions to deal with the fundamental concern of health. It is within the subject matter of anthropology to deal with sociocultural contexts of medical traditions and their practitioners and clients wherever they are found whether in so called western or non-western societies. However with its comparative perspective the anthropologists have been particularly interested to understand the conceptualization of illness and healing traditions in different parts of the world. In an applied context anthropological investigations can provide a most important insight into the way different medical traditions are institutionally embedded in particular societies and of the conditions (symbolic as well as material) which affect people's choices with regard to which health practitioner they seek out when illness strike.

The existence of several therapeutic traditions in a single cultural setting, is an especially important feature of medical care in the developing world (Leslie, 1978). Patients may feel uncertain as to what type of care provider can cure their illness, leading them to consult different medical therapists. Or they may decide that treatment of certain illness requires more than one type of assistance. Generally care is sought from several types of providers and medical traditions concurrently or sequentially. The practitioners of different medical traditions may interact with each other in a variety of ways - at times they borrow the ideas and knowledge from one another, compete with and oppose the other system, they may co-operate with one another developing referral systems or they may simply co-exist independently of one another (Kleinman, 1980: Levitt, 1988: Subedi, 1989).

The pluralistic character of health and medical system in almost every one society, be it simple or complex is being increasingly recognized (Minocha, 1980). This pluralism may receive state recognition and patronage, or the state may discourage it or remain neutral to its existence. 


\section{Understanding Medical Pluralism}

Although medical pluralism has been defined differently by different scholars, most of them have common understanding about it. Firstly, it may mean the coexistence of multiple traditions of medicine, including what are called folk sectors, popular sectors and professional sectors (Kleinman, 1980) which present multiple choices of therapeutic traditions to the individual (Durkin-Longley, 1984; Minocha, 1980). Kleinman (1980) developed a widely used model that recognizes three overlapping sectors in the health care traditions. The popular sector consists of health care conducted by ill persons themselves, their families, relatives, social network, and communities. It includes a wide variety of therapies, such as special diets, herbs, exercise, rest, baths, or over-the counter drugs. Kleinman, who has conducted research in Taiwan, estimates that $70 \%$ to $90 \%$ of the treatment episodes on that island occur in the popular sector. The folk sector encompasses healers of various sorts who function informally and often on a quasi-legal or sometimes, given local laws, an illegal basis. Examples include herbalists, midwives, mediums and magicians. The professional sector encompasses the practitioners and the bureaucracies of both biomedicine and professionalized heterodox medical traditions, such as ayurvedic and unani medicine in South Asia, herbal medicine and acupuncture in People's Republic of China. Each sector emphasizes the position of patients and healers within different clinical realities. Each type of healers in such a context typically attracts clients from a cross-section of society. Moreover, clients in these settings often try to seek around for therapy making use of more than one of the different therapeutic types available to them during a single illness episode, either simultaneously or in succession (Subedi, 1989).

Secondly, it may mean the choices within the particular tradition. An individual has not only a choice between consulting an ayurvedic practitioner or one practicing allopathic medicine, but within allopathic medicine, he has a choice to go the hospital (of a particular type) or to a doctor in a private clinic or health post, or medicine seller in a village or a town, in a nearby or a distant city. Then there is pluralism in the types of personnel who contribute to the practice of same medical tradition. Apart from specialists and other registered medical practitioners, there are other personnel, including nurses, technicians, dispensers and others who not only help the doctors but independently advise and work as medical persons (Dixit, 1999; Justice, 1986; Kristvik, 1999),

Extending the meaning of medical pluralism further, one may notice medical pluralism in people's conception of disease and illness, their resort to medical practices belonging to different systems, and their responses to other medical dimensions. For instance, inhabitants can categorize their illnesses as personalistic versus naturalistic (Foster and Anderson, 1978), natural versus supernatural (Devekota, 1984) dichotomy. Villagers resort to indigenous healers for supernatural causes of illness and to allopathic healers for natural causes of illnesses.

To this extended meaning of medical pluralism, one could add the pluralism among the medical practitioners themselves. There is considerable evidence that the general practitioner draws from varied systems in his medical practice. For example, an ayurvedic practitioner may incorporate the stethoscope, opthalmoscope and other instruments and drugs from modern medicine into his kit, and germ and virus theories in his explanatory armoury. Similarly, practitioners of the modern medicine may explain dietary restrictions in terms of hot-cold dichotomy of ayurveda (Minocha, 1980).

Medical Pluralism includes both the cognitive and social system aspects of healing and treatment traditions. The cognition dimension relates to a wide range of medical concepts, values, attitudes, and beliefs that serve as guidelines for health action and practices. Thus, it is easily possible that in one illness episode people have different theories of causality among the various medical traditions. The social system dimension refers to the different economic, institutional and organizational aspects of treatment and health-care delivery system. In looking at health care pluralism, wherever it occurs, it is an important to examine 
both the cognitive and social aspects of health care available to the individual patient.

\section{A Sketch of Nepali Medical Pluralism}

The daily life of health and healing in Nepal is comprised of a wide range of medical beliefs, knowledge and practices, and of distinctive categories of functionaries including medical doctors (specialized in allopathic medicine) ', health assistants, nurses, dispensing chemists and pharmacists, acupuncture therapists, Tibetan medical practitioners, ayurvedic ${ }^{2}$ practitioners, Unani medical practitioners ${ }^{3}$ folk healers, tantric healers, spiritual healers, dhami-jhankris (shamans), herbal doctors, traditional birth attendants, and other practitioners. Non formal or even illegal medical traditions are available as numerous alternative therapies. In the application of their medical knowledge, these doctors and healers use different

1 I use the term "allopathy" in this paper to be neutral. Although, here, I am not going to specify the terminological issues, different writers have used the words like "cosmopolitan", "western", "modern", "biomedicine" which is synonymous with angrezi (English) system. As Leslie (1976) notes, to call it "modern medicine" suggests a contrastive relationship to some conservative "traditional medicine; to call it "scientific medicine" suggests that other medical systems are unscientific. Similarly, to call it "western medicine" is to overlook its use in other parts of the world. However, these different words are used synonymously to refer to the allopathic medicine. Practitioners of this tradition in Nepal are called doctors (physicians and surgeons), health assistants, nurses, assistant nurse midwives, and so on.

2 Ayurveda, literally "the knowledge of life", refers to the vast body of ancient Hindu literature concerned with health and disease. The traditional practitioners in this tradition are usually called Kaviraj and vaidva in Nepal. Similarly, the word Vaidya refers to a wide variety of healers. A broad division can be made between those who stick exclusively to ayurvedic methods and diagnoses, and those who make use of an eclectic mix of ayurveda, astrology and trantric rituals. The latter are called jahrplutke vaidyas, that is, who use the technique jharmu (sweeping) and phuknu (blowing). Both ayurvedic practitioners and ritual healers are not sharply distinguished in local usage. However, medical graduates of ayurveda are now called doctors.

3 The Unani tradition of medicine came to Nepal through Islam and its traditional practitioners are usually referred to as hakims. Medical graduates of the Unani are also now called doctors. forms of diagnosis, therapy and medicines. They elaborate, develop and modify the old traditions and make new ones. Although a certain variation in medical knowledge, specialization and curing practices is found in almost all societies, in Nepal this variation is pervasive, and it may be the manifestations of cultural pluralism that characterizes many Asian civilizations.

The state funded health services, for example in Nepal, are also pluralistic in character. There is not only one type of health service in the country, nor is there uniformity among various health services. To name only a few, there are the Police and Army Hospitals preferred for them and their family, Tribhuvan University Teaching Hospital and other private or public Medical Colleges Hospitals, Mission Hospitals and numerous others. Within the Kathmandu Valley itself it is estimated that there are more than 350 agencies, government, private, missionary and community managed hospitals, health centers, health posts, sub health posts, and the others. Service charges, outdoor times and indoor facilities and specialties are different in each health institution.

Within the pluralistic medical setting, symptoms and associated causes of illness or health problems determine which practitioner one seeks initially for help and which treatment one expects or requests. However, a single illness may have completely different theories of causality among the various medical traditions. Therefore, it is not surprising that in one illness episode people may seek help from practitioners from more than one medical tradition until they are cured.

In order to understand the health care choice people make during their health complication episodes, it is important to recognize the various medical traditions, which influence people's medical decisions. In Nepal, the several medical traditions with their independent classification of illness, theories of disease causality, diagnostic methodologies, practitioners and treatment therapies present the health seeker with a wide menu of alternatives to choose from. Below are descriptions of three medical settings and their associated traditions. It should be noted that there are wide variations in practices and the beliefs 
among different ethnic groups found in the country. The following is a very simplified version of these systems to provide the reader with some sense of distinctions between them.

\section{Household Based Self Medication}

The concept of "household production of health" recognizes that a person's access to and use of various forms of treatment are connected to his or her social power in relation to other household members. Here, a "household" is examined rather than a "family", as a household may be easier to define. A household in this case may include, for example, other relatives living in the same house. Household productions of health examine how household members cooperate and compete for resource in order to restore, maintain and promote health. Caretakers attend to the patient through examinations, diagnosis and treatments.

Medical condition necessitates a variety of changes in a household. Indeed, the full extent of the resource and responsibilities, which need to be negotiated, may not be recognized by outside observers. These include fees for medications, analyses and examinations, transportation costs, arrangement for accompaniment, and loss of a worker and more likely of two workers either in wages of housework. All of these factors, and no doubt more, can influence heath-care seeking decisions. A consideration of the impact of the medical condition to the household helps explain why patients may come to the hospital too late for treatment.

As mentioned, a member's access to health care is often influenced by his or her position within the household. One member may receive immediate treatment or the more prestigious treatment, while another member may receive care only if the symptoms are prolonged or may receive less prestigious treatments, such as home remedies. Among the characteristics influencing a person's status within the household are his or her age, gender, generation, marital status and work status. Because of the variations in roles and responsibilities within a household, medical condition of various members affect the household differently. Since members' status within the household vary as does the impact of their illnesses, then their access to treatment also differs.

Further, a person's position within the household not only influences his/ her access to medical care, but also influences his/her sick role behavior. Thus a high status individual or wage earner may receive concern and medical attention over a cough, whereas a lower status individual may have to really moan in order to appear sick enough to receive attention. When an illness strikes, the afflicted person generally does not cope individually- whole network of supporting social ties are mobilized. The composition of members in this network is of course decided by the structural principles according to which right and obligations as well as emotional involvement tie individuals to each other in enduring groups.

In Nepal, the most important group is the patriarchal household (nuclear or joint). Although there is significant variations among households with regard to social, cultural and economic 'capital' (Bourdieu, 1977), one of their dominant concerns is biological reproduction. Socialization of its children and provision of livelihood for their members, households everywhere in Nepal, is the immediate group, which is mobilized when a disease strikes. Important stores of knowledge transmitted within households are brought into play when the health of its members is threatened. Exclusively households support the children, old and disabled members of the family.

Almost all households, at least at the initial stage of illness, utilize a fairly wide stock of intergenerationally transmitted as well as newly acquired knowledge and practices of healing to nurse the ill back to good health. The localized nature of society, limited access to, and relatively low quality of public health institutions and the prohibitive costs of allopathic medicine and modern health services also force most households to rely on home remedies which span from divination to faith healing and the use of local herbs. Increasingly, they also involve the use of off-the-counter allopathic drugs, which remain almost completely unregulated (NESAC, 1998). Using massage, administering herbs, manually removed obstructions, applying heat compresses and recommending dietary restrictions and 
prescriptions are the major self-medication practices in Nepal. Generally such medical problems are first treated at the home with some remedies suggested by relatives and neighbors. It is only after several attempts fail that a specialist is called in.

While access to the health post or hospitals are easier than in the past $(\mathrm{MoH}, 1997)$, home based system of remedies are playing an important role in health seeking behavior. For example, almost 90 percent of the childbirth in Nepal take place at home rather than in such facilities (NESAC, 1998). The rise and expansion of institutions for enhancement of individual-based knowledge (for example, hospitals and other health institutions) can be expected to change in its structure.

\section{Local Indigenous Medical Traditions}

In general, when the home remedy to cure an ill person fails, a larger net of intervening agents are brought in, namely community level healers. They may vary among and within religions; ethnic groups and locally they operate without any government recognition of formal coordination. Their medical traditions and practices may be based on religion and beliefs regarding cosmos, ideas about cultural construction of persons, personal relationships between humans and the environment, and diet. Healers often specialize in particular techniques and which specialist is consulted depends on the nature of illness itself.

In terms of religion one's health or ill health can be attributed to one's relationship with the spirit of world of antigods (demons) and gods (whether one has satisfied or angered them), the relationship between the deeds of one's past lives and one's present life's predestination (fate), and one's deeds according to Hindu and Buddhist scripture (virtuous or sinful (Hitchock and Jones 1976; Dietrich 1998; Miller 1979). In this tradition one explains illness in terms of dharma (religion) or karma (fate), prevents illness by properly attending to ritual, making promises to the gods, giving offerings and doing sacrifice, through prayer, and by following the scripture. Illness is diagnosed and cured by jyotisis (astrologers), guru-purohits (priests), or monks through prayers and rituals.
The magical spirit world affects health and can cause illnesses through the network of angry ghosts (spirits of persons who have died in violent or other unnatural deaths), monster-like bhut-prets (spirits), angry gods and anti-gods, and bokshis (witches-person who can cast evil spells by performing inverted religious rituals). Illness in this tradition is believed to be caused by "supernatural attacks (lagu, lagu-bhagu) resulting from jealousy (especially in the case of witches) or one's having ignored or offended a spirit. Attacks can be in the form of possession or some type of physical harm. Specialists who help to diagnose and cure illness brought on by such attacks are commonly referred to as faith healers including sorcerers (those who can cast and break spells and have the ability to communicate with the spirit world), shamans (those who go into spirit possession trances, can perform exorcism and can act on the behalf of spirits), and mediums or jannemanche (those who can act as transmitters between the spirit world and the non spirit world). One study (reference year 1977) estimated the number of various categories of faith healers between 400,000 to 800,000 in Nepal (Shrestha and Lediard, 1980) for a population of $\mathrm{Ca} .13$ million people, which roughly translates one faith healer for every six households. The number of doctors at the same time was 500, and the number of nurses 334 (Shrestha and Lediard, 1980:34). This figure can itself be taken as an indication of the legitimacy of the tradition. Different research findings (for example, Okada, 1976, Durkin-Longley, 1984, Gellner, 1994) show that even next to the hospital in Kathmandu Valley, traditional healers are many, and in high demand. Diagnosis is done by going into trance, divination, and by inquiring about and observing physical symptoms. Prevention of attack is done through rituals, by providing the individual with an amulet, by being careful not offend spirits or persons suspected of being witches, by keeping a fire lit, by being cautious of persons seen at night and by staying away from haunted places. Treatment is done by chanting mantras and shouting at the spirits to leave the person's body, violent exorcisms by which the patient is burned, frightened and beaten until the spirits flee the patient's body, and by giving offerings and doing sacrifices. The significant role of 
local healers has been widely noted (Blustain, 1976, Okada, 1976; Wake, 1976, Stone, 1976) in different parts of Nepal. The majority of sick persons in the rural area, who eventually visit the allopathic healers, consult the traditional healers first as the hierarchy of resort.

\section{Ayurvedic, Homeopathic and Unani Traditions}

The ayurvedic medicine is based on the classical Sanskrit medical tradition of 'Ayurveda' (literally "science of life"). This tradition of healing has been practiced in South Asia since ancient times. It bases itself on a well-developed system of physiological characteristics of the ill person, symptoms of illness and detailed pharmacological knowledge of herbs and their processing techniques.

Within the ayurvedic medical tradition a person's body is composed of prakriti (the female component of the cosmos which forms the body) and three dosas (humors) (wind, bile, and phlegm) which are responsible for all bodily processes. The equilibrium of the dosas maintains health and imbalance results in ill health. In this tradition, diseases or disorders are caused by physiological imbalances resulting from poor food habits, environmental changes, and shock to the system. Diagnosis is done by pulse reading, physical examination, and appraisal of symptoms. Disorders are treated with herbal medicines, diet control, lifestyle control, surgery, meditation, and changing one's environment. Many Nepalese believe in its efficacy in the long run, that is, as capable of achieving more permanent healing with no harmful side effects.

The government of Nepal has formally recognized this medical tradition in Nepal. There are several governmentaffiliated institutions of ayurveda. For example, there is an ayurvedic campus in Kathmandu under the Institute of Medicine, Tribhuvan University, and the Department of Ayurveda in the Ministry of Health, and some recognized health centers and pharmacies. Some ayurvedic practitioners have their own private clinics. Ayurvedic doctors attend the medical schools either in Nepal or in India for a degree in Ayurvedic Medicine and Surgery. As early as the mid-eighties it had been estimated that there were 1500-2000 ayurvedic practitioners in Nepal and 113 government ayurvedic dispensaries (Streenfland, 1985). Most ayurvedic healers in Nepal work within the private domain. Recently, ayurvedic healing in the public sector is performed through one central ayurvedic hospital with 50 beds, one 15-bed zonal hospital, 172, dispensaries in 55 districts, and a central drug-manufacturing unit $(\mathrm{MoH}, 1997)$. However, fewer resources are devoted to them than to allopathic medicine.

Homeopathy was introduced in Nepal as early as 1920 as a natural healing system. Homeopathic healing is largely a private sector initiative, which encompasses approximately 500 practitioners and 100 clinics (MoH 1997). Within the public sector, there is only one homeopathic facility with hospitalization facilities for six patients.

The unani healing tradition, with preventive, promotive and curative services, has an extremely limited reach. In addition, the Tibetan healing tradition, acupuncture therapy, Japanese healing cults (Seimeiko), and naturopathy are also practiced in the selected areas of the country.

\section{Allopathic Medical Tradition}

This tradition is based on the germ theory of disease and studies of anatomy and physiology. The worldview that has shaped the allopathic medicine is commonly referred to as the mechanistic paradigm ${ }^{4}$. The characteristic of this mechanistic paradigm is the separation of the component part and emphasis on the detail study of it. According to this tradition illhealth, manifested by various signs and symptoms, results from pathological processes in the biochemical function of the body. In this medical tradition health and normality are defined by reference to certain physical and biochemical parameters, such as weight, height, blood pressure, heart rate or visual activity. For each measurement there is a numerical range-the normal value-

4 The mechanistic paradigm describes the notion of the body as a machine. disease as the consequence of the breakdown of the machine, and the doctor's task as a repairer to the machine. 
within which the individuals are normal and healthy. Above or below these ranges are abnormal condition. This model is the conceptual tool for the analysis of the bodily problem for allopathic medical practitioners in Nepal.

In this tradition, medical treatment is based on the theory that a disease can be carried from person to person, through the air, the blood or bodily secretions or from contact with a septic object or substance. Officially, the allopathic medicine was introduced in Nepal at the end of the nineteenth century when the Bir Hospital was established in Kathmandu. Organized development of allopathic services started in mid-1950s. This system is formally associated with the public health care delivery in Nepal through the Ministry of Health and a number of private offices staffed by physicians, public health professionals, nurses, midwives, health assistants, pharmacists, village health workers, and trained volunteers. Diagnosis are made by the physical examinations, appraising symptoms, taking blood pressure, doing the laboratory test of blood, urine and stool, taking the body temperature, taking X-ray, and doing cathscans. Treatments are given through the injections, medicines, blood transfusion, surgery, and electric shock, bed-rest, recommending changes in behaviors or diets, and physiotherapy. Prevention of diseases and illnesses are done through immunizations, health education and promotion, birth control, dietary restrictions, and an early examination and screening tests. Nepali people have a considerable faith in the technology of allopathic medicine, particularly in injections and antibiotics (Gellner, 1994). A wide range of allopathic medicine manufactured in India and Nepal are freely available without an authorized person's prescriptions. The in-charge of medicine sellers at medical halls (so called pharmacies) who lack any formal training are selling the "prescription only" drugs.

Allopathic medical facilities, pharmaceuticals, and trained personnel, however, are neither reliable nor easily available in rural areas. Almost fifty percent of the country's doctors, most sophisticated and large private nursing homes and hospitals, trained medical professionals, and the health facilities are concentrated in Kathmandu Valley. Government-run health services provide care unevenly in the countryside; it is remote and inconvenient for allopathic practitioners (Macfarlane, 1994; Pigg, 1995). Maintaining a supply of drugs to remote health posts is a constant problem, as is keeping health posts and district hospitals staffed with trained, active practitioners (Justice, 1986).

In my view, Biomedicine must be seen in the context of capitalist world system. Some of the particular agents of the world system operating in the biomedical sector include international health agencies, foundations, national bilateral aid programs, all multinationals (especially drug firms, medical technology producers and suppliers, polluting and exploiting industrial firms, agribusinesses, commercial baby food suppliers, purveyors of chemical fertilizers and pesticides, and seller of population control devices), and a medical cultural hegemony supportive of the activities of these agents on the world scene and in particular nations and locales (Elling, 1981 quoted in Baer et al., 1997). At all levels the state care systems of advanced capitalist nations reproduce the structure of the class relations. The profit making orientation caused allopathic medicine to evolve into a capital-intensive endeavor heavily oriented to high technology, the massive use of drugs and the concentration of services in medical complexes. The state legitimizes the corporate involvement in the health arena and reinforces it through support for medical training and research (Baer et al., 1997).

There a re a number of tendencies that are the characteristics of allopathic medical tradition in Nepal. The first tendency is that the locations of medical hospitals and research institutions, allocation of resources are centralized in urban areas. The largest, most prestigious, most specialized and most money consuming curative institutions are located in the large urban centers (Streefland, 1985; Subedi, 2001b). The same applies to the location of institutions where medical knowledge is developed, stored and taught. It is difficult to find medical schools, libraries and research centers outside the larger towns. The politicians and administrators who steer and take decisions regarding the health policy of the country stay in Kathmandu, the 
capital city. Similarly, the drugs to be used are generally manufactured in the urban centers or in the rich countries. The ruling elites that control country collaborate with international agencies, foundations and bilateral aid program to determine health policies (Justice, 1986). These elites and the agents they deal with often advocate nationalize and preventive medicine, but their actions favor curative rather than preventative approaches to health care for themselves and even for the lower social strata. This situation clearly shows that the further away people live from the center in physical and / or social distance, the more difficult it becomes to get good quality and sufficient quantity of medical supplies, facilities and staffs (Streefland, 1985). This holds for a peripheral village and for an urban slum. Although for the urban poor health-care institutions and medicines are at a reasonable distance, the costly nature of allopathic medicine plays an important role for not consulting the allopathic medical practitioners.

The second tendency of the allopathic medicine is that in the developing countries like Nepal, allopathic medicine is capitalist and commercial in orientation. Many curative institutions, pharmaceutical companies and medical equipment industries are privately owned. The goal of many medical practitioners is to work privately and earn more money. Profit making is an important consideration in the delivery of health care, and production and sale of drugs and materials. The consequence of such factors is that, broadly speaking, the best services and facilities are available in those places where most people are living and where most wealth is concentrated. For the rural villages it means relatively low numbers of drug sellers and relatively unqualified medical practitioners (Subedi, 2001b). On the other hand, the large demand for curative services create an excellent environment for the activities of unqualified medical practitioners and unlicensed drug sellers.

\section{Factors Influencing Development and Acceptance of Medical} Traditions

Different medical traditions in Nepal are co-existing very well. Each tradition has own expertise, especially in certain illnesses. In many cases, the question of which specialist to consult depends on the nature of illness. For example, most of the people seek ayurvedic vaidya or Kaviraj for jaundice, and dhami-jhankri, janne manche, jharphuke vaidya or other local healers for lagu or evil spirits. Minor discomfort, wounds and sores are often treated at homes with foods and herbs; more serious injuries, the hospitals, clinics or health post (allopathic healers) are consulted. But except for this, the larger pattern is not a matter of who consult when, but whom one consult first and why.

People's actual health behavior in situation offering choices is the outcome of the weighing process. Various factors are known to be taken into account. One such factor is the perception which villagers have of health care personnel. This study, has, for instance, made clear that people's performance largely explains why certain healers who belong to the same village and relate to their clients also as kinsmen, neighbors or afno manche are often more popular than the healers from outside. Other determining factors are the cost of treatment in relation to what people can afford and to the quality of service they will get. The actual cost of using the services, those of reaching the services, the opportunity cost (loss of money which could have been earned in the same time) all are important in this respect.

\section{Cultural and Historical Forces}

People's understanding of the bodily affliction and searching for cure is one of the most powerful forces in maintaining the continued acceptance of any medical traditions. The members of each cultural group have deep rooted beliefs that their own tradition must be useful if not the ideal system for handling the illness recognized by the culture. Many informants say that allopathic medical practitioners have no curative treatment for illnesses caused by ancestors, bokshi caused illnesses, Ajima and other types of lagu illnesses. According to them the jhakphuke or jannemanche do have such treatments. In particular, since the illhealth is defined some what differently from culture to culture, and certain diseases state reputedly due to supernatural causes including phut-pret and bokshi, are therefore recognized only by its own members. These types of understandings serve to reinforce the value of the particular medical tradition. Some culture bound syndromes, such as sarko, 
janaikai, bokshi bigar play an important role to continue the existing medical traditions. In the contexts of each culture, certain illness phenomena, particularly in relation to psychological illhealth, are best explained and dealt with within the cultural context of its concept of the universe. Where the cultural concept differs very substantially between the sick individual and the therapist, this will be extremely difficult. Many informants think that they should try to find different types of healers within as well as between the traditions simultaneously, altering or sequentially in the hope that one, if not all, will eventually rid him of their illness.

Most of the patient's version on the relative performance of different healers was varied according to the nature of the culturally perceived diseases and their expertise. For instance, patients with constipation, jaundice or respiratory problems preferred ayurvedic healers due to long term and durable action whereas allopathic drugs relieved them temporarily. According to them, allopathic remedies were mostly effective on accidental injuries. Likewise, trantric healers or jharphuke vaidya are perceived more effective against lagu and bokshi bigar. After they consult the varieties of healers with a range of remedial actions, their experience with the relative performances compel them to feel, perceive and value those healers they employed.

\section{Economic Forces}

Cost and accessibility of the medical care is an important factor in influencing the choice of a tradition of medical care. In the developing countries like Nepal, where the bulk of people live in poor economic conditions, the cost of specialist medical doctors' fees, pathological test, and medicines are usually beyond their means. The government expenditure on health is generally low. The large numbers of people living in the village remains beyond the reach of this medical sector. When illnesses are minor, most people in Kathmandu Valley seek local healers or take over-the-counter, easily accessible and affordable medicines. Consequently, people often have to depend upon locally available medical facilities which are within their geographical and economic reach. In such areas, locally available healers and their medications are only forms of cares that are within the economic means of people.

\section{Influence of Other Medical Traditions}

If the dominant medical tradition is unable to provide adequately for the care of the population, other medical traditions fill these gaps. If a patient uses a variety of remedy but is unable not recover, he tries to find the alternative therapies or cure the disease. They adapt the behavioral aspect of medical tradition without fully grasping and accepting the theory behind them. They may, in other words, seek them for the purely pragmatic reason that they perceive it as being effective. For example, acupuncture, as an alternative medical tradition became accessible to them via different sources of informants. Most of the patients responded that they approached acupuncture clinics as the final attempt before the death. They were frustrated with a painful life with chronic sufferings. They have spent thousands of rupees and visited a number of medical specialists in the country and "why not once go or experience acupuncture?" were responses of many people.

Finally, procedures employed for diagnosis, treatment or cure in different health care practitioners is closely related to the performance of efficacy. Besides, knowledge and value perceived by healers and patients on physical, mental or spiritual concerns, healers behaviors play the decisive roles, whether patients seek them or not. For example, patients during discussion commented that they prefer kind-hearted, smiling or cheerful, friendly healers who listen to their sufferings. This way, patients search for relatively expert, senior and experienced health professionals. As most of the patient's view, remedy in any way from which could recover them quickly is desirable. And if the remedy reacted with harmful side or after effects they seek other healers, which could help them, recover without such side effects or after effects.

\section{Interaction of Medical Traditions}

These different medical traditions do not exist as closed cultural systems. Their practitioners interact with one another in 
a variety of ways, and at times they borrow from one another, compete with and oppose each other. They may cooperate with each other in moving patients through particular referral systems. Through such interaction there may develop a certain integration of elements of . knowledge from different traditions. Other elements in their traditions and practices may remain insulated and co-exist independently of one another. For example, traditional healers may give modern medicine, faith healers and traditional birth attendants are being trained as village health volunteers and community health leaders, physicians prefer ayurvedic medicine for hepatitis, local ayurvedic healers or herbalists after being unsuccessful in curing a patient refer patients to the hospitals, whereas spiritual healers or shamans condemn beliefs about ghosts and witches as superstitious. Durkin-Longley (1984), in a study of urban Nepal, shows that specific illness ideally are brought to practitioners of one medical traditions; Jaundice (kamalpitta) is brought to the ayurvedic doctor, mental illness to the dhami-jhankris and jharphuke vaidyas, and accidents to the modern (allopathic) medical practitioners.

Not all systems exist to an equal extent in all parts of Nepal. The local indigenous system exists to a far greater extent in rural areas than either the modern medical or ayurvedic systems do. However, wherever one is in Nepal, there is a pluralistic medical setting in which people must make their health care choice.

\section{Concluding Remarks}

A number of studies in Nepal have shown that persons seek different types of healers based on their perception and beliefs regarding the illness problem, which in turn are influenced and defined by their social surroundings and network of relationship. The most widely prevailing medical system in Nepal is faith healing. The fatalistic nature of people play a distinct role in Nepali society, especially when someone in the family suffers from chronic illness, mental illness, or is not able to have even a single child. Hence faith healers or shamans such as Dhami, Jhankri, Lama, Guruwa and the like receive wide public acceptance and play a significant role in meeting the health-care need of the villagers. The patients in the hilly regions of Nepal are more likely to contact "dhami-jhankri" first than other health-care providers.

Most patients in Nepal use home remedies and delay seeking professional help. These remedies included the herbal remedies and foods to eat / avoid. If the problem continues, the next resort is a traditional healer. The modern health care services are only sought as a last resort, usually for the serious and persistent problems. This finding also shows that patients who do seek treatment at health facilities use both traditional and modern medicine according to their perception of effectiveness.

The point here is that treatment decisions in medically pluralistic settings typical of the developing countries like Nepal are complex. The choice of healers is shaped by a wide range of factors, among them perceptions of efficacy, practical considerations (such as distance), symbolic considerations, the perceived cause of the ailment and whether it is viewed as life threatening, and personal attributes of the patient. This body of research shows that the existence of biomedicine is only as a treatment option. The presence of alternative sources of health care can significantly effect the choice of health care services. It is apparent from this paper that anthropological knowledge and research method have not yet realized their full potential for contributing to contemporary health issues. Thus, in assessing health care service utilization, various factors associated with medical pluralism need to be considered seriously.

\section{REFERENCES}

Baer, Hans A. et al. (1997). Medical Anthropology and the World System. Bergin and Gravey, Westport.

Barth, Fredrik (1993). Balinese Worlds. The University of Chicago Press, Chicago.

.....(1990). "The Guru and the Conjurer: Transactions in Knowledge and the Shaping of Culture in South-east Asia and Malanesia." Man 25: 640-653.

Blustain, Harvey S. (1976). "Levels of Medicine in a Center Nepali Village." Contributions to Nepalese Studies. Center for Nepal 
and Asian Studies, Tribhuvan University, Kathmandu, Nepal. Special issue, Vol.3,

Bourdieu, Pierre (1977). Outline of a Theory of Practice (Translated by Richard Nice). Cambridge University Press, Cambridge.

Christakis, N icholas A. et al.(1994). Illness Behaviour and Health Transition in the Developing World. In Lincoln C. Chen (ed.) Health and Social Change in International Perspective. Harvard Series on Population and International Health, Harvard, University Press.

Devekota, Padam Lal (1984). Illness Interpretation and Modes of Treatment in Kirtipur. Contributions to Nepalese Studies. Centre for Nepal and Asian Studies, Tribhuvan University, Kathmandu, Nepal Vol.11, No. 2 Pp11-20.

Dietrich, Angela (1998). Tantric Healing in Kathmandu Valley: A Comparative Study of Hindu and Buddhist Spiritual Healing Traditions in Urban Nepalese Society. Book Faith India, Delhi.

Dixit, Hemang (1999). The Quest for Health. Educational Enterprise (P) Ltd, Kathmandu.

Durkin-Longley, Maureen (1984)."Multiple Therapeutic Uses in Urban Nepal." Social Science and Medicine. Vol. 19, No, 8, Pp 867. 872.

..(1988)."Ayurvedic Treatment for Jaundice in Nepal." Social Science and Medicine. Vol. 27, No. 5, Pp 491-495.

Elias, Norbert (1991)."On Human Beings and Their Emotions: A Process-Sociological Essay." In Mike Featherstone et al, (Ed.) The Body: Social Process and Cultural Theory. SAGE Publications Ltd, London.

Foster, George and Anderson, Barbara (1978).Medical Anthropology. New York: John Wiley and Sons, Inc.

Foster, George M. (1994).Hippocrates' Latin American Legacy: Humoral Medicine in the New World. Gordon and Breach Science Publishers.

Geertz, Clifford (1973). The Interpretation of Culture. New York: Basic Books.

Gellner, David N.(1994). "Priest, Healers, Mediums and Witches: The Context of Possession in Kathmandu Valley, Nepal." Journal of Royal Anthropological Institute, Vol. 29, No.1, PP. 27-48.

Goffman, Erving (1959). The Presentation of Self in Everyday Life. Doubleday Anchor Books, Doubleday \& Company, New York.
Good, Byron J. (1994). Medicine, Rationality and Experience: An Anthropological Perspective. Cambridge University Press.

Helman, Cecil G. (1996). Culture, Health and Illness: An Introduction for Health Professionals (third Edition). ButterworthHeinemann, Oxford.

Hitchock, John T. And Jones, Rex (Ed) (1976). Spirit Possession in the Nepal Himalayas. New Delhi: Vikas Publishing House.

Justice, Justice (1986). Policies, Plans, and People: Culture and Health Development in Nepal. University of California Press, Berkeley.

Kleinman, Arthur (1978). Concepts and Model for the Comparison of Medical Systems as Cultural Systems. Social Science and Medicine, Vol.12 Pp 85-93.

(1980). Patients and Healers in the Context of Culture. Berkeley: University of California Press.

Kristvik, Ellen (1999). Drum and Syringes. Bibliotheca Himalayica, Series III, Volume VII

Leslie, Charles (1978). "Pluralism and Integration in the Chinese Medical System." In A. Kleinman et al. (Ed.) Culture and Healing in Asian Societies. Cambridge, MA: Schenkman Publishing Company.

......(1980). Medical Pluralism in World Perspective. Social Science and Medicine. 14B, 4: 191-195.

Levitt, Marta (1988). From Sickle to Scissors: Birth, Traditional Birth Attendants and Perinatal Health Development in Rural Nepal. Unpublished Ph. D. Thesis, University of Hawai.

Lieban, Richard W. (1977). "The Field of Medical Anthropology." In David Landy (Ed.) Culture, Disease and Healing: Studies in Medical Anthropology. Macmillion Publishing Co., Inc. New York.

Minocha, Aneeta (1980). "Medical Pluralism and Health Services in India." Social Science and Medicine. Vol. 14B Pp 217-223.

Miller, Casper J. (1979). Faith Healers in the Himalayas. Centre for Nepal and Asian Studies, Kathmandu.

MoH (1997). Nepal family Health Survey. Family Planning Division, Ministry of Health.

NESAC (1998). Nepal Human Development Report 1998

Okada, Ferdinand (1976). "Notes on two Shamans-curers in Kathmandu. "Contributions to Nepalese Studies. Center for Nepal and Asian Studies, Tribhuvan University, Kathmandu, Nepal.Special issue, Vol.3, Pp 107-112. 
Pelto, Pertii and Pelto, Gretel (1996). "Research Design in Medical Anthropology." In Sargent Carolyn F. and Johnson, Thomas M. (Ed.) Medical Anthropology: Contemporary Theory and Methods. Praeger Publishers.

Pigg, Stacy Leigh (1989). Here, There and Every Where: Place and Person in Nepalese Explanations of Illness. Himalayan, Research Bulletin. Vol. ix, NO. 2 Pp 16-23.

.....(1995). "Acronyms and Effacement: Traditional Medical Practitioners (TMP) in Internal Health Development." Social Science and Medicine. Vol. 41, No. 1, Pp 47-68.

Scheper-Hughes, Nancy and Margret, Luck (1998). "The Mindful Body: A Prolegomenon to Future Work in Medical Anthropology." In Peter J. Brown (Ed.) Understanding and Applying Medical Anthropology. Mayfield Publishing Company.

Shrestha, R.M. and Lediard M. (1980). Faith Healers: A Force for Change. Kathmandu, UNFPA / UNICEF.

Stone, Linda (1976). "Concept of Illness and curing in a central Nepal village." Contributions to Nepalese Studies. Center for Nepal and Asian Studies, Tribhuvan University, Kathmandu, Nepal. Special issue, Vol.3, Pp 55-80

Streefland, Pieter (1985). "The Frontier of Modern Western Medicine in Nepal." Social Science and Medicine. Vol. 20, No. 11, Pp $1151-1159$.

Subedi, Janardan (1989). "Modern Health Services and Health Care Behaviour: A Survey in Kathmandu, Nepal." Journal of Health and Social Behaviour. Vol. 30, (December), Pp 412 420.

........ (1988). Factors Affecting the Use of Medicine in a Pluralistic Health Care System: The Case of Nepal. Unpublished Ph. D. Thesis, University of Akron.

Subedi, Madhusudan Sharma (2001a). Medical Anthropology of Nepal. Udaya Books, Kathmandu, Nepal. .(2001b). Development and Underdevelopment of Modern Health Services in Nepal. Deva-Vani, Devaghat Vedic Adhyatmic Parisad, Kathmandu. Vol. 4 pp 217-224

Wake, C.J. (1976). Health Services and Some Cultural Factors in Urban Nepal. Contributions to Nepalese Studies. Center for Nepal and Asian Studies, Tribhuvan University, Kathmandu, Nepal. Special issue, Vol.3, Pp 113-125. 